\title{
O conceito de simetria e o ensino de Álgebra: analisando materiais curriculares da Educação Básica
}

\section{Bárbara Passadore de Oliveira}

Universidade Federal do ABC (UFABC)

babizzi@gmail.com

\author{
Alessandro Jacques Ribeiro \\ Universidade Federal do ABC (UFABC) \\ alessandro.ribeiro@ufabc.edu.br
}

\section{Arthur B Powell}

Rutgers University-Newark

powellAB@andromeda.rutgers.edu

\begin{abstract}
Resumo
Fundamentado em uma pesquisa de mestrado que teve por objetivo compreender como o conceito de simetria se manifesta na Álgebra escolar, o presente artigo se propõe a apresentar e a discutir os principais resultados do referido estudo. A pesquisa foi desenvolvida tomando-se por objeto de análise materiais curriculares elaborados para a Educação Básica. Utilizando-se da análise de conteúdo como ferramenta metodológica, identificaram-se poucas manifestações do conceito de simetria relacionado em situações de ensino voltadas para o campo da Álgebra. Destaca-se, entre os resultados, a potencialidade de contemplar o conceito de simetria quando do estudo da função polinomial do segundo grau, possibilitando uma articulação entre a Geometria e a Álgebra, ao tomar as relações entre as raízes da função e o eixo de simetria e as propriedades da parábola.
\end{abstract}

Palavras-chave: Simetria. Ensino de Álgebra. Análise Documental. Materiais Curriculares.

\section{The concept of symmetry and teaching of Algebra: analyzing curricular materials from Basic Education}

\begin{abstract}
Based on a master's degree research that aimed to understand how the concept of symmetry is manifested in the school algebra, this paper presents and discusses the study's main findings. The research was based on an analysis of curricular documents prepared for elementary and secondary education. Using content analysis as a analytic tool, we identified in the documents manifestations of the concept of symmetry in school algebra. A main finding concerns the potential of contemplating the concept of symmetry when studying the roots of univariate second-degree polynomial functions, the axis of symmetry, and other properties of parabolas. Such a study enables links between geometry and algebra.
\end{abstract}

Keywords: Symmetry. School Algebra. Document Analysis. Curricular Materials. 


\section{Introdução}

O presente artigo propõe-se a apresentar alguns resultados da dissertação de mestrado de Oliveira (2015), inserida no projeto "Conhecimento matemático para o ensino de Álgebra: uma abordagem baseada em perfis conceituais", vinculado ao Programa Observatório da Educação (Obeduc), financiado pela Coordenadoria de Aperfeiçoamento de Pessoal do Ensino Superior (Capes).

A referida pesquisa de mestrado ficou circunscrita às regiões de inquérito da Educação Algébrica e da Formação de Professores de Matemática, fundamentada no referencial teórico elaborado por Ball e seus colaboradores (BALL; THAMES; PHELPS, 2008) e em pesquisas que discutiam diferentes significados de conceitos matemáticos, como o de equação (RIBEIRO, 2013). Do ponto de vista da Matemática, encontramos, na obra Uma história da simetria da Matemática (STEWART, 2007), uma possibilidade de estabelecer articulação entre Álgebra e geometria por meio do "conceito de simetria". Desse modo, fundamentados na abordagem algébrica dada ao conceito de simetria no trabalho de Stewart, o objetivo de nossa pesquisa se configurou em identificar e analisar as potencialidades deste conceito, no campo da Álgebra, em documentos oficiais e na proposta curricular do estado de São Paulo.

Apresentado o cenário no qual foi realizada a presente pesquisa de mestrado que gerou este artigo, passamos à constituição de nossa revisão de literatura, a qual contempla os fundamentos teóricos de nosso estudo. Em seguida, trazemos a metodologia e os procedimentos metodológicos que sustentaram a pesquisa, a apresentação e as análises dos dados. Por fim, finalizamos nosso artigo com as conclusões, as considerações e os encaminhamentos futuros.

\section{Revisão de literatura: das pesquisas exploradas aos fundamentos teóricos de nosso estudo ${ }^{1}$}

Feitos o levantamento e o mapeamento da literatura nacional e internacional, foi possível compreender com mais profundidade nosso problema de pesquisa: desvelar o papel do conceito de simetria no âmbito da Álgebra Escolar. Com isso, nossa busca passou, então, de maneira mais direcionada, à identificação e à compreensão das possíveis potencialidades do conceito de simetria para o campo da Álgebra e para o modo como tal conceito aparecia articulado a essa grande área ou inserido nela.

\footnotetext{
${ }^{1}$ Neste artigo nosso referencial teórico concentrou-se na fundamentação matemática do conceito de simetria e suas relações/implicações com/na Álgebra. Na pesquisa de mestrado que originou este texto, no entanto, tomamos por unidade de análise a dimensão do conhecimento profissional docente nos documentos analisados. Para tal, utilizamos o conceito de "Conhecimento Matemático para o Ensino" (no original, MKT, Mathematical Knowledge for Teaching). Tais discussões podem ser encontradas em Oliveira (2015).
} 
Tomando por base nossa revisão de literatura, observamos que a problemática em torno do conceito de simetria está, quase sempre, inserida no contexto do ensino de geometria, sobretudo em aspectos ligados às transformações geométricas. Não encontramos evidências, nas pesquisas por nós revisadas, de que o conceito de simetria seja abordado no âmbito da Álgebra escolar (PAVANELLO, 1989; PASSOS, 2000; PEREIRA, 2001), ainda que tenham sido encontradas diversas manifestações deste conceito - simetria - no âmbito da Álgebra superior (FARMER, 1999).

Tendo em vista nosso problema de pesquisa e os encaminhamentos dados a seu desenvolvimento, consideramos oportuno discutir os fundamentos teóricos que sustentaram nosso estudo, utilizando os pontos de vista de dois eixos condutores, quer sejam: o conceito de simetria e o conceito de conhecimento matemático para o ensino.

Tomando por base referenciais específicos da área da Matemática, encontramos, na obra Grupos e simetria (FARMER, 1999), uma importante discussão na qual o autor associa o conceito de simetria a propriedades geométricas de uma figura. Ele aponta, em sua obra, o que é a simetria de uma figura, quais tipos de simetria existem; e mostra as combinações de simetrias em diversos polígonos. Tais resultados foram considerados em nosso estudo, na medida em que nos auxiliam a identificar e a compreender manifestações do conceito de simetria no campo da Álgebra.

Um importante resultado apresentado por Farmer (1999), dentre esses aspectos citados anteriormente, é obtido a partir do estudo das simetrias do quadrado. O autor determina o conjunto das simetrias do quadrado e percebe que a estrutura desse conjunto é equivalente à estrutura de grupos. A partir disso, Farmer desenvolve a generalização de que o conjunto das simetrias de qualquer polígono será um grupo. Com isso, fundamentado nas discussões do autor, identificamos aqui, uma evidência da manifestação da simetria na Álgebra, uma vez que a estrutura de grupo é uma das estruturas algébricas.

Um outro espaço no qual fomos buscar inspirações para nossas inquietações foi a História da Matemática. Dentre as manifestações do conceito de simetria que encontramos ao longo da história, destacamos uma vertente na Álgebra. E, para isso, trazemos uma breve discussão acerca de como o conceito de simetria se situa no cerne do desenvolvimento da Teoria de Grupos, de Galois, no que se refere às propriedades das equações algébricas e às propriedades dos grupos.

Segundo Brandemberg (2010), uma das mais importantes constatações de Galois acabou por estabelecer uma condição para que uma equação fosse solúvel em relação a seu grau. Em continuidade a seus trabalhos, Galois preocupou-se em identificar em quais casos uma equação é solúvel por radicais (no caso, equações de grau primo), o que lhe permitiu concluir que há uma condição de grupo e subgrupos formados com as raízes, a qual possibilita ou não sua solubilidade 
por radicais. Assim, a estrutura do grupo, segundo Galois, de uma equação geral de grau 5 não possui solubilidade por radicais.

Considerando as discussões até aqui apresentadas, nosso trabalho buscou investigar se e como o conceito de simetria aparece em documentos oficiais e em manuais do professor, pois identificar e compreender como este tema está disposto nesses documentos pode nos auxiliar a entender o que o professor poderá contemplar em suas aulas acerca do conceito de simetria.

Foram escolhidos para nossa análise os documentos oficiais: os Parâmetros Curriculares Nacionais e a Proposta Curricular do Estado de São Paulo. Além disso, no intuito de investigar o currículo matemático dos estudantes, será feita a análise do Caderno do Professor do estado de São Paulo.

\section{Metodologia: do contexto de nossa pesquisa aos procedimentos metodológicos}

Com base no que foi apresentado em nosso referencial teórico e em nossa revisão de literatura, entendemos que a abordagem qualitativa é a mais adequada aos nossos propósitos. Além disso, cabe situar nosso estudo dentro da perspectiva de uma pesquisa bibliográfica (GIL, 2010).

No âmbito da Educação Matemática, tomamos por base o trabalho de Stylianides e Ball (2004). A partir desses autores, desenvolvemos nossas escolhas metodológicas para investigar o conceito de simetria do ponto de vista do ensino de matemática na Educação Básica. Os autores consideram seis diferentes estratégias metodológicas para pesquisas que investigam o conhecimento matemático para o ensino, dentre as quais, utilizamos as estratégias Análise dos documentos oficiais e Análise do currículo matemático dos estudantes, que nos serviram para nortear os encaminhamentos da pesquisa. Escolhemos essas duas abordagens porque, no que tange à pesquisa bibliográfica, acreditamos que os documentos oficiais poderiam nos orientar a respeito do que é considerado importante para o ensino de simetria na Matemática Escolar. Buscamos compreender, nesses materiais, quais as indicações e as orientações acerca do ensino do conceito de simetria e da forma como ele tem sido exigido dos alunos.

Uma vez explicitadas as fontes bibliográficas de nossa pesquisa, optamos pela análise de conteúdo como método de analítico em nosso estudo. Tomamos por base os pressupostos teóricometodológicos apresentados por Bardin (2011). Há de se destacar que, em nossa pesquisa, não tomamos a análise de conteúdo como uma simples indexação do material analisado. Ratificando o que Bardin (2011, p. 48) esclarece, tomamo-la como um conjunto de análise que consiste na “manipulação de mensagens (conteúdo e expressão desse conteúdo) para evidenciar indicadores que permitam inferir sobre uma outra realidade que não a da mensagem”. 
Assim sendo, passamos a identificar e a analisar qual o caráter da abordagem dada ao conceito de simetria nos documentos e nos materiais selecionados, ou seja, de que forma e com qual frequência esse tema aparece relacionado com a Álgebra e/ou com os conceitos algébricos. Com base nisso, pudemos compreender como o conceito de simetria é entendido e contemplado na educação básica e o que se espera que os alunos entendam desse conteúdo.

\section{Nossas análises: o conceito de simetria e a matemática escolar}

Buscamos organizar nossas análises em duas principais etapas: (i) a primeira, que denominamos de Análises de primeiro nível, consistiu principalmente na leitura flutuante, com a identificação de itens de significado, e na formulação de hipóteses que foram responsáveis por nortear a segunda etapa; (ii) a segunda, denominada por nós como Análises de segundo nível, retoma as unidades de significado para averiguar a quais contextos pertenciam e para conferir quais dessas manifestações poderiam ser entendidas como simetria na Álgebra escolar.

As análises de primeiro nível do material selecionado buscaram identificar as Unidades de Codificação (Unidades de Registro - UR e Unidades de Contexto - UC). Essa indexação ocorreu, $a$ priori, durante a leitura flutuante da Proposta Curricular do Estado de São Paulo, dos Parâmetros Curriculares Nacionais (PCN) Ensino Fundamental II, dos Parâmetros Curriculares Nacionais Ensino Médio (PCNEM) e dos PCN+ (Orientações complementares aos PCN do Ensino Médio). Concomitantemente à identificação das UC, ocorreu a formulação de hipóteses que serviram de guias para nossas análises.

Iniciamos pelos Parâmetros Curriculares Nacionais dos Terceiro e Quarto Ciclos do Ensino Fundamental. No bloco denominado "Espaço e forma", entre os conteúdos de geometria, aferimos 15 ocorrências da UR "simetria" ou "simetrias". Com isso estabelecemos as seguintes hipóteses: (i) Os parâmetros curriculares estabelecem a simetria como pertencente ao campo da geometria. Sendo assim, entendemos que é provável que o Caderno do Professor siga a mesma linha; (ii) A relação direta das UR "simetria" ou "simetrias" com os conteúdos e os conceitos de Álgebra não foi encontrada, mas imaginamos possível que haja essa manifestação nos eixos temáticos desenvolvidos no Caderno do Professor.

Em seguida desenvolvemos a análise dos Parâmetros Curriculares do Ensino Médio (PCNEM) e dos Parâmetros Curriculares do Ensino Médio+ (PCN+). Os PCNEM apresentam as competências e as habilidades a serem desenvolvidas em Matemática, porém, como não há orientações/indicações de qualquer conceito matemático em particular explicitado, não conseguimos identificar nenhuma ocorrência das UR "simetria" e "simetrias". 
Por fim, passamos à análise da Proposta Curricular do Estado de São Paulo. A primeira UR identificada foi "simetrias", a qual se dá na descrição do bloco temático "geometria". Logo em seguida, identificamos apenas uma ocorrência da UR "simetria", a qual estava localizada no Caderno do $7^{\circ}$ ano, $2^{\circ}$ bimestre, também dentro do bloco "geometria". Essas análises nos permitiram formular hipóteses: (i) as ocorrências das UR "simetria" ou "simetrias" estarão ligadas majoritariamente com os conceitos de geometria; (ii) a Proposta Curricular, por ser um documento abrangente e pouco específico, não apresenta qualquer alusão a relações entre as UR "simetria" ou "simetrias" com a Álgebra; (iii) há conteúdos que, de acordo com a revisão de literatura, podem ser promissores para uma articulação entre as UR "simetria" ou "simetrias" com a Álgebra.

Dando continuidade à leitura flutuante, fizemos uma busca horizontal na versão do professor dos "Cadernos" dos Anos Finais do Ensino Fundamental e do Ensino Médio. Nosso intuito nesse momento era apenas verificar a quantidade de ocorrências (UR), ignorando o contexto em que elas ocorrem ou ao qual estão relacionadas. No volume III $\left(6^{\circ}\right.$ ano $\left.{ }^{2}\right)$, identificamos 34 ocorrências dentro do eixo "Espaço e Forma"; portanto, todas as ocorrências identificadas aqui se correlacionam com tópicos da geometria. Nenhuma das UR encontradas fez alusão à Álgebra. Ao analisar o volume I ( $7^{\circ}$ ano), identificamos apenas uma ocorrência da UR "simetria", a qual estava associada aos números inteiros; ela é, portanto, a primeira ocorrência de tal UR não inserida em temas ou conteúdos da geometria ou, ainda, relacionados ao eixo "Espaço e Forma". Continuando nossas análises, agora no volume II ( $7^{\circ}$ ano), observamos a ocorrência de 65 manifestações das UR "simetria" ou "simetrias", todas elas relacionadas a temas da geometria. De nossas análises no volume III ( $8^{\circ}$ ano), depreendemos a presença de duas ocorrências das UR "simetria" e "simetrias", ambas, mais uma vez, imersas no campo da geometria. Por outro lado, ao analisar o volume II ( $9^{\circ}$ ano), apesar de identificarmos apenas uma ocorrência da UR "simetria", esta ocorreu dentro de um tema da Álgebra - equação de segundo grau - e relacionada com a UR “eixo".

Passando às análises dos exemplares do "Caderno do Professor" para as séries do Ensino Médio, começamos com o volume II ( $1^{\mathrm{a}}$ série), no qual são identificadas 17 ocorrências das UR "simetria" e "simetrias", totalmente evidenciadas dentro de um conceito algébrico: o conceito de função polinomial do segundo grau. Nas análises do volume III ( $1^{\text {a }}$ série $)$ localizamos apenas uma ocorrência da UR "simetria”, a qual se encontra relacionada à UR "percepção", quando se discutia o plano cartesiano e se abordava a percepção da relação de simetria na bissetriz dos quadrantes ímpares. Ainda que não apareça claramente, o Caderno se refere à bissetriz como um eixo de simetria do plano cartesiano. O volume I ( $2^{\mathrm{a}}$ série) aborda conceitos de trigonometria, mais

\footnotetext{
${ }^{2}$ Iremos utilizar a nomenclatura atual para se referir aos "anos finais do ensino fundamental"
} 
especificamente a circunferência trigonométrica, e ali sete ocorrências das UR foram identificadas, todas elas associadas exclusivamente a conceitos geométricos. As duas ocorrências da UR "simetria" no volume I ( $3^{\mathrm{a}}$ série) estão evidenciadas dentro do capítulo "Geometria Analítica" e não ocorrem imersas no campo da Álgebra; e, por fim, ao analisar o volume IV ( $3^{\text {a }}$ série), identificamos quatro ocorrências da UR "simetria", todas inseridas no tema "Estatística".

Nesta segunda parte de nossas análises, quando desenvolvemos as análises de segundo nível, buscamos estabelecer correlações com a literatura e com nosso referencial teórico. Pretendemos agora elucidar aos leitores quais hipóteses, formuladas inicialmente em nossa pesquisa, foram refutadas ou ratificadas ao longo de nosso estudo, com o intuito de encontrar potencialidades ou manifestações do conceito de simetria dentro de temas matemáticos pertencentes ao campo da Álgebra.

Iniciamos nossas análises com os PCN do Ensino Fundamental. Ali foram obtidas 15 ocorrências das UR "simetria" ou "simetrias", tendo havido, dentre elas, apenas três ocorrências situadas não no eixo "Espaço e Forma", mas, sim, no eixo "Tratamento da Informação". Inicialmente havíamos conjecturado que, mesmo inserido em um eixo dos PCN que não fosse o de Espaço e Forma, o conceito de simetria estaria vinculado a conceitos da geometria. Dessa forma, a partir de nossas análises, pudemos então ratificar nossa hipótese, uma vez que esse documento coloca a simetria como uma propriedade geométrica dos objetos matemáticos em questão, sem ênfase ou enfoque algum em aspectos algébricos de tal conceito.

Ao analisar a Proposta Curricular do Estado de São Paulo, identificamos apenas duas ocorrências das UR "simetria" ou "simetrias" dentro do bloco geometria, fato que foi fundamental para a formulação de certas hipóteses a respeito de Caderno do Professor, o qual contemplaria o conceito de simetria imerso na geometria. Isso também nos possibilitou conjecturar em quais tópicos poderiam aparecer certas potencialidades para explorar o conceito de simetria do ponto de vista da Álgebra. Tal assertiva que levantamos até o momento, sem dúvida, se fundamenta e se apoia na literatura por nós estudada, principalmente pelas considerações discutidas em nosso referencial teórico (FARMER, 1999; STEWART, 2007).

Em seguida nossas análises de segundo nível foram realizadas no Caderno do Professor. Voltamos nosso olhar agora para todas as ocorrências e as relações das UR "simetria" e "simetrias" que possam estar vinculadas aos temas da Álgebra, buscando apresentar Unidades de Contexto (UC) para justificar, elucidar e ilustrar as relações por nós identificadas, sempre tomando-se por base e referência as considerações e os resultados apresentados anteriormente, quando da apresentação de nosso referencial teórico (FARMER, 1999; STEWART, 2007). 
O primeiro volume que apresenta a ocorrência da UR "simetria" imersa no campo da Álgebra é o volume I ( $7^{\circ}$ ano), no eixo "Números e Operações". Aqui, entendemos que o conceito de simetria está não só imerso na Álgebra, mas em função da Álgebra, ou seja, sua manifestação ocorre dentro de um conceito algébrico - no caso, a estrutura dos números inteiros. Além disso, tal conceito também se direciona para a produção de conhecimentos na Álgebra, o entendimento sobre características e as propriedades dos números.

Uma outra ocorrência da UR simetria no campo da Álgebra ocorre agora no volume II ( $9^{\circ}$ ano). Nesse material é proposta uma atividade contextualizada que apresenta uma função polinomial do segundo grau, seguida de seu gráfico para o estudo da dependência das variáveis; da identificação do ponto de máximo; e dos valores simétricos em relação ao eixo de simetria que passa pelo vértice da parábola. No entanto, apesar da ocorrência identificada, ao analisar o contexto no qual a situação está imersa, consideramos que essa passagem não preconiza a produção do conhecimento na Álgebra, pois o material, na verdade, utiliza a simetria como uma propriedade geométrica da parábola (simetria axial), sem se aprofundar, por exemplo, nas implicações algébricas dessa propriedade (por exemplo relações dos zeros da função e sua localização em relação ao eixo de simetria da parábola).

Nossas análises de segundo nível seguem para um outro volume (o da primeira série do Ensino Médio), no qual verificamos 18 ocorrências das UR "simetria" ou "simetrias". A primeira delas ressalta o conceito de simetria como um conceito a ser estudado, designado como um dos "fatos fundamentais relativos às funções de $2^{\circ}$ grau" (SÃO PAULO, 2013a, p. 38). Nessa parte do material também estão descritos: gráficos, intersecção com os eixos, coordenadas do vértice e estudo dos sinais.

Esse estudo dos gráficos segue ao longo do material por nós analisado, contemplando quase todas as ocorrências de "simetria" ou "simetrias" identificadas nele. As duas próximas ocorrências a serem analisadas não só estão imersas na Álgebra, como também utilizam o conceito de simetria para a produção do conhecimento dentro do estudo do gráfico de uma função. $\mathrm{O}$ material apresenta relações entre as coordenadas dos pontos de uma parábola a partir da posição de seu eixo de simetria. Tal verificação pode ser evidenciada por meio desta UC:

Se o eixo de simetria for o próprio eixo $y$, então para cada valor de $y$ correspondem dois valores de $x$ com sinais opostos: $(\mathrm{a}, \mathrm{y})$ e $(-\mathrm{a}, \mathrm{y})$. Se o eixo de simetria estiver deslocado horizontalmente de $h$ unidades, então os pontos equidistantes terão coordenadas $(\mathrm{a}+\mathrm{h}$; y) e (-a + h; y). (SÃO PAULO, 2013a, p. 40)

Em seguida, são identificadas duas outras ocorrências da UR "simetria" no material, as quais aparecem como legendas de figuras que objetivam ilustrar visualmente, para o aluno, a propriedade de a parábola ser uma curva que possui um eixo de simetria cujas raízes podem ser 
estabelecidas a partir do deslocamento desse eixo em relação ao eixo y. Podemos observar tais análises na Figura 1.

Figura 1 - Eixo de simetria da parábola
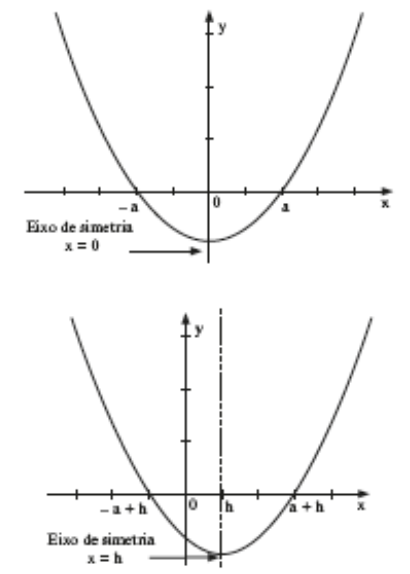

Fonte: São Paulo (2013a, p. 40)

A ocorrência da próxima UR também apresenta uma propriedade das parábolas, a qual relaciona o vértice com o eixo de simetria. Nesse trecho, o Caderno apresenta algumas inferências em relação ao valor das raízes e ao vértice da parábola, porém sem explicitar a simetria como o conceito responsável por sustentar matematicamente tal relação. Em nosso entendimento, isso mostra que, mais uma vez, o conceito de simetria aparece por um viés geométrico (principalmente no que se refere ao caráter visual e à percepção), não tendo sido devidamente exploradas suas potencialidades para o ensino da Álgebra. Tal relação poderia ser explorada no campo da Álgebra e vai ao encontro do que é contemplado nos trabalhos de Stewart (2007) e Farmer (1999).

Em continuação às análises, a próxima ocorrência retoma o que é o eixo de simetria de uma parábola. Ao apresentar uma tabela de valores para serem descobertos, é sugerido ao professor “comentar com os alunos os resultados obtidos que refletem a ideia de simetria na parábola" (SÃO PAULO, 2013a, p. 41). Parece-nos evidente, nesse trecho, que o Caderno do professor apresenta o conceito de simetria imerso na Álgebra, uma vez que se utiliza dessa propriedade para sugerir o aprofundamento do estudo da função polinomial do segundo grau.

Outra ocorrência no material analisado se encontra na sugestão de resolução da seguinte situação: “Quais são as coordenadas do ponto V, vértice da parábola?” (SÃO PAULO, 2013a, p. 42). Para ilustrar a abordagem algébrica dada à simetria, temos a seguinte UC: "Em razão da simetria do gráfico, concluímos que o vértice e o ponto médio do segmento do eixo $\mathbf{x}$ está entre 0 e 4, ou seja, no vértice temos $x=2 "$ (SÃO PAULO, 2013a, p. 42). Nesse trecho, o Caderno do Professor apresenta claramente a simetria como base para a construção do conhecimento em Álgebra, pois utiliza a propriedade de simetria do gráfico para inferir o valor da coordenada dos 
pontos. Tal possibilidade já tinha sido apresentada por nós, anteriormente, como um importante articulação entre o que estamos analisando e as discussões e implicações de nosso referencial teórico para o estudo do conceito de simetria dentro dos domínios da Álgebra.

Outro trecho do material analisado que entendemos ser importante colocar em discussão aponta também que, por meio do eixo de simetria, podemos descobrir as coordenadas do vértice da parábola. Isso pode ser evidenciado na UC descrita a seguir:

Para determinar as coordenadas do vértice da parábola, podemos proceder do seguinte modo: sabemos que $\mathrm{f}(0)=\mathrm{c}$; em razão da simetria do gráfico, existe outro valor de $\mathbf{x}$ tal que $\mathrm{f}(\mathrm{x})=\mathrm{c}$; calculando, obtemos: $\mathrm{ax}^{2}+\mathrm{bx}+\mathrm{c}=\mathrm{c}$ acarreta $\mathrm{ax} 2+\mathrm{bx}$ $=0$, de onde tiramos $\mathrm{x}=0$ ou $\mathrm{x}=-\mathrm{b} / \mathrm{a}$. (SÃO PAULO, 2013a, p. 46)

Identificamos outra ocorrência nesse Caderno: "No presente Caderno, optou-se pela apresentação da fórmula que explicita diretamente a simetria das raízes em relação ao valor de $\mathrm{x}_{\mathrm{v}}$ " (SÃO PAULO, 2013a, p. 51). Ali se apresenta o conceito de simetria como base para a "fórmula de Bháskara"; assim, nessa passagem, em nossa interpretação, também fica evidente uma forte potencialidade do conceito de simetria para a construção do conhecimento na Álgebra.

A última discussão relevante que observamos é no momento em que o Caderno do Professor coloca a importância de estabelecer a relação inversa entre as funções exponencial e logarítmica, em vista do eixo de simetria da bissetriz dos quadrantes ímpares. Contudo, por outro lado, o Caderno não apresenta nenhuma explicação ou aprofundamento dessa questão. Nesse momento, o próprio material traz uma abordagem algébrica do conceito de simetria, mas sem explicitá-la, nem aprofundá-la, de modo a contribuir para que professor e aluno construam o conhecimento algébrico a partir dessa ideia.

Com o intuito de sistematizar e apresentar nossos dados e nossas análises referentes ao Caderno do Professor, no que se refere às ocorrências das UR "simetria" e "simetrias", elaboramos o esquema exposto na Figura 2.

Figura 2 - Síntese dos dados obtidos
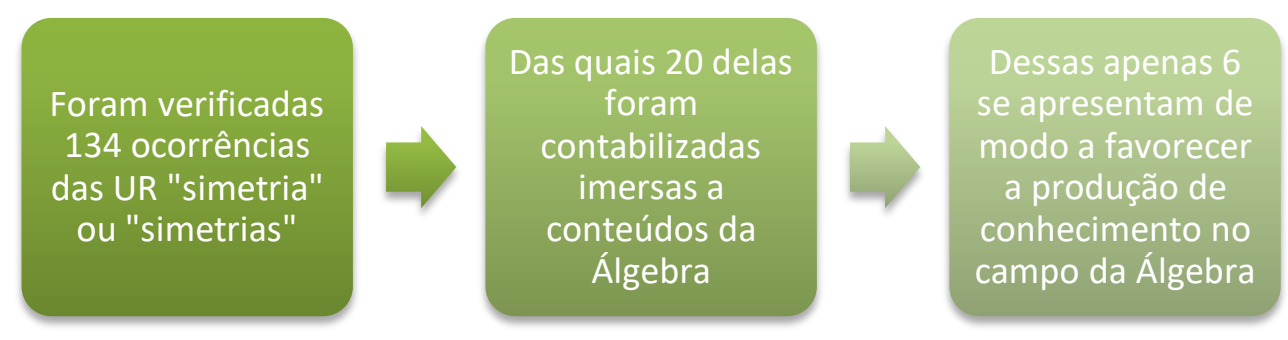

Fonte: Elaborada pelos autores

Entendemos o conceito de simetria como promissor para a produção do conhecimento no campo da Álgebra. Tal conclusão está fundamentada nas diversas evidências anteriormente 
discutidas, nas quais são apresentadas as UR "simetria" ou "simetrias" em diferentes contextos matemáticos, muitos deles imersos em conceitos da Álgebra ou relacionados a eles. Tais evidências, certamente, foram identificadas por nós em nossas análises, graças aos fundamentos teóricos proporcionados pelos trabalhos de Farmer (1999) e Stewart (2007).

Assim, retomamos aqui nossas hipóteses formuladas no início da análise do material, que apontavam para uma possível escassez do conceito de simetria no campo da Álgebra. A despeito de não termos verificado, de fato, uma frequência alta de ocorrências das UR analisadas no campo mencionado, as ocorrências das UR na Álgebra, em função da produção de conhecimento nesse campo são significativas para ratificar a potencialidade do conceito de simetria para o desenvolvimento de temas da Álgebra. Por outro lado, em nossas análises, observamos situações em que, mesmo identificando a ocorrência de uma UR imersa em um conceito ou tema da Álgebra, isso não necessariamente indica produção de conhecimento nesse campo da Matemática.

Finalmente, entendemos que nossas análises nos permitem concluir que há pouca relação entre o conceito de simetria e a Álgebra nos materiais didáticos e nos documentos curriculares da Educação Básica, constatação já esperada, tendo em vista as pesquisas por nós estudadas em nossa revisão de literatura. Além disso, imaginamos termos conseguido, ao menos em parte, apresentar potencialidades de conexão e exploração do conceito de simetria em temas matemáticos relacionados à Álgebra, de forma implícita e explícita, como apresentado em todas ocorrências das UR e das UC ao longo das análises. Faremos algumas outras conexões na próxima secção, quando apresentaremos nossas considerações finais.

\section{Considerações finais}

Encerramos nosso artigo, colocando destaque em aspectos referentes às ocorrências das UR no campo da Álgebra e ao estabelecimento de UC para tais inserções. No que se refere aos documentos curriculares, apresentamos um panorama que entendemos ser fundamental para ratificar as impressões obtidas, no escasso material didático e/ou bibliográfico, que abordam o conceito de simetria na Álgebra. Nossos resultados ratificaram o que já havia sido constatado em nossa revisão de literatura e que, inclusive, nos levou à formulação de hipóteses que foram verificadas em nossas análises.

No tocante aos Cadernos do Professor, com a análise dos dados, pudemos estabelecer dois importantes resultados: o primeiro atesta que a simetria, no que diz respeito à Álgebra escolar, parece-nos fundamental para o entendimento do conceito de função polinomial do segundo grau, seja por meio da representação do gráfico em forma de parábola, seja por meio da representação da equação e da fórmula de "Bháskara" para resolução. Isso é evidenciado, uma vez que o material 
didático analisado propõe diversas relações entre as raízes da função e o eixo de simetria, assim como sugere um estudo da "fórmula de Bháskara" sob o olhar do eixo de simetria. Destacamos mais uma vez a importância que nossa fundamentação teórica exerceu na "abertura de nossos olhos" para potencialidades de contemplar o conceito de simetria quando esse se relaciona - direta ou indiretamente - com conceitos do campo da Álgebra.

Uma vez compreendida essa abordagem do conceito de simetria no campo da Álgebra, voltemo-nos para o segundo resultado. Parece-nos legítimo notar que a potencialidade de tal abordagem, quando explorada dentro de conceitos e temas da Álgebra, é palco de uma rica interlocução entre a Álgebra e a Geometria, permitindo ao aluno estabelecer fortes relações e inferências no estudo de determinado tópico matemático, de um ponto de vista mais amplo e geral. Tais relações entre a Álgebra e a Geometria, seja por meio do conceito de simetria - como bem nos lembra os trabalhos de Farmer (1999) e Stewart (2007) - seja por meio de outros conceitos matemáticos, parece-nos fundamental para um trabalho integrado entre essas diferentes áreas da Matemática e, ainda, para um trabalho interdisciplinar, com a Física, por exemplo.

Portanto, diante de nossa análise e dos resultados obtidos, deixamos aqui nossa inquietação em relação à necessidade latente - e, para nós, evidente - de investigar, segundo Stylianides e Ball (2004), como o conceito de simetria é contemplado em outros documentos e fontes de dados como o currículo dos professores, o conhecimento matemático dos professores, o conhecimento matemático dos estudantes, e nas práticas escolares da matemática.

Por fim, consideramos que os resultados obtidos aqui ainda podem ser ampliados, aprofundados e ramificados em outras pesquisas no campo da Educação Matemática. Esperamos que o presente trabalho tenha convidado os leitores a uma reflexão a respeito de oportunidades e potencialidades de uma nova abordagem para o ensino e aprendizagem de Matemática, no que se refere a interlocuções entre a Álgebra e a Geometria e tenha, também, elucidado nossas pretensões, impressões e os resultados obtidos com essa pesquisa.

\section{Referências}

BALL, D. L.; THAMES, M. H.; PHELPS, G. Content knowledge for teaching: What makes it special? Journal of Teacher Education, East Lansing, v. 59, n. 5, p. 388-407, 2008.

BARDIN, L. Análise de conteúdo. Tradução Luís Antero Reto e Augusto Pinheiro. São Paulo: Edições 70, 2011.

BRANDEMBERG, J. C. Uma análise histórico-epistemológica do conceito de grupo. São Paulo: Livraria da Física, 2010.

BRASIL. Ministério da Educação e do Desporto. Secretaria de Educação Fundamental.

Parâmetros Curriculares Nacionais: Ensino Fundamental. Brasília: MEC/SEF, 1998. 
BRASIL. Ministério da Educação e do Desporto. Secretaria da Educação Fundamental.

Parâmetros Curriculares Nacionais. Ensino Médio. Brasília: MEC/SEF, 2000.

FARMER, D. W. Grupos e simetria: um guia para descobrir a Matemática. Lisboa: Gradiva, 1999.

GIL, A. C. Como elaborar um projeto de pesquisa. São Paulo: Atlas, 2010.

OLIVEIRA, B. P. de. O conceito de simetria na álgebra escolar: um estudo baseado na análise de documentos oficiais e manuais de professores. Dissertação (Mestrado em Ensino, História e Filosofia das Ciências e Matemática) - Universidade Federal do ABC, Santo André, 2015. 76ff.

PASSOS, C. L. Representações, Interpretações e Prática Pedagógica: a Geometria na Sala de Aula. Tese de Doutorado. Universidade Estadual de Campinas. Faculdade de Educação de Educação. Campinas, 2000.

PAVANELLO, R. M. O abandono da geometria: uma visão histórica. Dissertação (Mestrado em Psicologia Educacional). Universidade Estadual de Campinas, Campinas, 1989.

PEREIRA, M. R. O. A geometria escolar: uma análise dos estudos sobre o seu abandono.

Dissertação de Mestrado. Pontifícia Universidade Católica. São Paulo, 2001.

RIBEIRO, A. J. Elaborando um perfil conceitual de equação: desdobramentos para o ensino e a aprendizagem de matemática. Ciência \& Educação, Bauru, v. 19, n. 1, p. 55-71, 2013.

SÃO PAULO. Caderno do professor: Matemática. Ensino Fundamental e Médio. São Paulo: SEE, 2013a.

SÃO PAUlO. Proposta Curricular do Estado de São Paulo. Ensino Fundamental e Médio. São Paulo: Secretaria da Educação, 2013b.

SHULMAN, L. S. Those who understand: knowledge growth in the teaching. Educational Researcher, Washington, v. 15, n. 2, p. 4-14, 1986.

STEWART, I. Uma história da simetria na Matemática. Rio de Janeiro: Zahar, 2007.

STYLIANIDES A. J.; BALL D. L. Studying the mathematical knowledge needed for teaching: The case of teachers' knowledge of reasoning and proof. Educational Research Association, San Diego, v. 5, p. 201-208, 2004. 